\title{
Response of an infinite beam resting on the tensionless Winkler foundation subjected to an axial and a transverse concentrated loads
}

\author{
Yin Zhang ${ }^{\mathrm{a}, \mathrm{b}, *}$, Xiaoming $\operatorname{Liu}^{\mathrm{a}, \mathrm{b}}$ \\ ${ }^{a}$ State Key Laboratory of Nonlinear Mechanics (LNM), Institute of Mechanics, Chinese Academy of Sciences, Beijing 100190, China \\ ${ }^{\mathrm{b}}$ School of Engineering Science, University of Chinese Academy of Sciences, Beijing 100049, China
}

\section{A R T I C L E I N F O}

\section{Keywords:}

Beam

Winkler foundation

Tensionless foundation

Closed form solution

\begin{abstract}
A B S T R A C T
The presence of an axial load changes the system stiffness of an infinite beam on a tensionless Winkler foundation, which also determines the solution form of the beam deflection. Five different closed form solutions depending on the axial load are analytically derived in this study. The beam deflections are systematically studied when it is subjected to various axial and transverse concentrated loads. Because the contact zone of a tensionless contact is not known a priori, it can cause a significant mathematical difficulty in solving various tensionless contact problems. In recent tensionless contact researches, the major efforts have been developing the efficient but complex algorithms to determine the contact zones. Five transcendental equations governing the contact zones, which are straightforward for numerical computations, are also analytically derived. The five closed form solutions can serve as a guide line for the further study of the beam tensionless contacts with more complex loading scenarios. Furthermore, the model of a beam on the Winkler foundation subjected to an axial load is also demonstrated to be applicable to various and different problems.
\end{abstract}

\section{Introduction}

The tensionless contact is variously called receding contact (Keer et al., 1972), unbonded contact (Weitsman, 1969), unilateral contact (Dempsey et al., 1984) and one-way contact (Attar et al., 2016), which all emphasize its asymmetric property of contact. The tensionless foundation as indicated by its name reacts to compression only. As a flexural structure can bend upwards and thus separate from a tensionless foundation, the foundation reaction force depends on the sign of displacement. At least two different governing equations, which are connected by the transverse conditions at the separation points (Kerr, 1976), are needed for the contact and non-contact zones. From the mechanics point of view, this causes a discontinuity of the contact constitutive law, which mathematically introduces the non-smoothness characteristics and nonlinearity into the problem (Attar et al., 2016). Unlike the geometrical nonlinearity of an elastic foundation (Ansari et al., 2010, 2011), this non-smoothness induced nonlinearity causes a significant difficulty in the tensionless contact problems. As a result, the key problem in the tensionless contact is to determine the contact zone (s). Recent studies on tensionless contact have been focusing on developing more general and efficient methods of finding the contact zones for the complex loading scenarios (Ma et al., 2009a, 2009b;
Nobili, 2012, 2013), the nonlinear foundation or foundation with several parameters (Nobili, 2012, 2013; Sapountzakis and Kampitsis, 2011a, 2011b, 2013) and dynamics (Bhattiprolu et al., 2014; 2016; Attar et al., 2016). Almost all studies have to develop complex algorithms to solve the beam tensionless contact problems. Few exceptions are, for example, the Weitsman's (1970) analytical solution to an infinite beam resting on the tensionless Winkler foundation, and Noblili's (2013) Green function method on a finite beam resting on both the tensionless Winkler and Pasternak foundations. Weitsman's (1970) analytical solution has been serving as a cornerstone for various studies on the beam tensionless contact, such as a finite beam under an asymmetric loading or with a gap distance between the beam and foundation (Zhang and Murphy, 2004, 2013), or under two concentrated loads (Nobili, 2013), or resting on the Reissner foundation (Zhang, 2008), etc. Furthermore, Weitsman's (1970) analytical solution is also a benchmark solution to validate the newly developed methods or algorithms on the beam tensionless contact (Nobili, 2013; Zhang and Murphy, 2004, 2013). However, Weitsman's (1970) analytical solution is for the zero axial load case only. The other four cases are presented with the closed form solutions in this study. The closed form solutions provide a much simpler and more efficient way of studying the beam tensionless contact than most of the algorithms.

\footnotetext{
" Corresponding author. State Key Laboratory of Nonlinear Mechanics (LNM), Institute of Mechanics, Chinese Academy of Sciences, Beijing 100190, China. Tel.: +861082543970 .

E-mail address: zhangyin@lnm.imech.ac.cn (Y. Zhang).
} 
Historically, the study on the beam tensionless contact is closely related with the stress and stability analyses of railway tracks (Chen and Chen, 2011; Choros and Adams, 1979; Kerr, 1974; Kerr and El-Aini, 1978; Lancioni and Lenci, 2010; Lin and Adams, 1987; Maheshwari et al., 2004; Tsai and Westmann, 1967). Because of the bending deflection induced by the track-wheel contact (Choros and Adams, 1979; Lin and Adams, 1987) or the vertical buckling due to thermal stress (Kerr, 1974; Kerr and El-Aini, 1978), the railway track can separate from its ballast. Because the ballast is an elastic supporting layer consisting of crushed stones, it cannot exert tensile forces on the track. As a result, the tensionless foundation model captures the track behavior more accurately than the foundation model that reacts to both tension and compression (Lin and Adams, 1987). Besides the crushed stones, a lot of supporting materials, such as soil (Lancioni and Lenci, 2010; Maheshwari et al., 2004; Sapountzakis and Kampitsis, 2011a, 2011b, 2013), foam (Bhattiprolu et al., 2014; 2016), ice (Kerr, 1972) and concrete (Wright, 1995), etc, all demonstrate the unilateral property of reacting to compression only. For many years, the track has been the main cause of limiting the operating speed of trains (Kerr, 1974). Thermal stress (Kerr, 1972, 1974; Labra, 1975), train moving load (Hetényi, 1946; Timoshenko and Langer, 1932; Weitsman, 1971) and moving mass (Ang and Dai, 2013; Dimitrovová, 2017; Tran et al., 2014) all effectively exert a compressive axial load on a track, which can cause the track instability and thus affect the safety of trains. A large number of the train accidents are due to the track instability (Lim et al., 2003). The continuous welded rail (CWR), which can be several kilometers long (Lim et al., 2003), is widely used in modern railways. Because of the complete elimination of expansion joints in the CWR tracks (Kerr, 1974; Lim et al., 2003), the thermal stresses due to the varying temperature can cause considerable axial compression or tension. In fact, the possibility of the track buckling due to thermal stress was the main reason for delaying the use of CWR track by decades (Kerr, 1974). Although Timoshenko and Langer's (1932) earlier work concluded that the dynamic effect on the track stress analysis is very small and a static analysis is thus sufficient, they implicitly assumed that the train speed is very low (Kerr, 1972). Because the effective compressive axial load is proportional to the square of the train speed for both the moving load and moving mass models, the dynamic effect must be considered in the analysis of the high-speed train-track interactions (Ang and Dai, 2013; Dimitrovová, 2017; Tran et al., 2014).

Besides modeling the actual axial load, incorporating an axial load into the model of a beam resting on the Winkler foundation is mathematically equivalent to introducing a new foundation model with one more parameter, which can better characterize an elastic continuum. An elastic foundation model in essence assumes the local response, i.e., the foundation reaction at a given point is determined only by its displacement, curvature and other higher order derivatives at that point. With this assumption, the foundation reaction can be incorporated into the differential governing equation of a structure, such as beam, plate and shell (Kerr, 1964). In other words, the elastic foundation model provides a differential formulation for contact problems, which also retains the mathematical simplicity (Kerr, 1964; Reissner, 1958). If the elasticity theory is used to analyze the contact between a structure and an elastic continuum modeled as the elastic half-space (Weitsman, 1969, 1972), the Boussinesq problem with integral or integrodifferential formulation has to be solved (Johnson, 1985). Physically, the integral or integrodifferential formulation indicates that the response of a given point in an elastic continuum is nonlocal, which depends on the responses of all other points. Mathematically, the integral or integrodifferential formulation is much more difficult than a differential one. Furthermore, for a high-speed railway support consisting of six layers of different materials (Bian et al., 2014), the elasticity analysis will be extremely difficult and complex if not impossible. The Winkler foundation is the simplest foundation model with only a spring layer of one parameter, which more or less causes some deviations from the response of a realistic material (Kerr, 1964). To obtain the physically close and mathematically simple representation of a realistic material, there are two main approaches: One is to introduce some assumptions in the elasticity analysis of a continuum to simplify the Boussinesq problem. For example, the assumption of the shear force independence on the depth leads to the Reissner foundation model (Reissner, 1958). The other is to add more layer(s)/parameter(s) to the Winkler foundation. For example, the Filonenko-Borodich foundation and the Pasternak foundation (Kerr, 1964) add a membrane layer and a shear layer onto the spring layer of Winkler foundation, respectively.

This study shows that under various axial loads, the contact zone of an infinite beam on the tensionless foundation is independent on the transverse concentrated load. This independence property leads to a very useful result: For the track-wheel contact with different concentrated loads, only one computation is needed. On the other hand, our solution indicates that the beam deflection in the non-contact zone will increase linearly to infinity as the distance from the concentrated load locus increases. Weitsman (1970) did not give the beam deflection solution to the non-contact zone and the discussion on the beam deflection unphysically reaching infinity is thus not presented. In this study, we show that this unphysical solution actually satisfies the equilibrium as described by the model. The unphysical problem can be corrected by adding a distributed load of the beam/track self-weight (Tsai and Westmann, 1967; Weitsman, 1970). However, with the presence of a distributed load, the above independence property is no longer valid. The computation has to be taken case by case because the contact zone depends on the magnitudes of both the concentrated and the distributed loads (Tsai and Westmann, 1967). In the modeling aspect, the loading scenario with a single concentrated force is a limit case of the general loading scenario with both concentrated and distributed loads (Tsai and Westmann, 1967). In the railway application, the concentrated load due to the track-wheel contact is much larger than the track self-weight. Therefore, the solutions to the beam resting on the tensionless foundation subjected to an axial and a concentrated loads can be an efficient tool of evaluating the track deflection and other related problems.

\section{Model development}

As shown in Fig. 1(a), the governing equation for a beam on the tensionless Winkler foundation subjected to an axial load $T$ and a transverse concentrated load $P$ is given as the following:

$$
\left\{\begin{array}{l}
E I \frac{d^{4} y_{1}}{d x^{4}}-T \frac{d^{2} y_{1}}{d x^{2}}+k y_{1}=P \delta(x),|x| \leq x_{o}, y_{1} \geq 0, \\
E I \frac{d^{4} y_{2}}{d x^{4}}-T \frac{d^{2} y_{2}}{d x^{2}}=0,|x| \geq x_{o}, y_{2} \leq 0,
\end{array}\right.
$$

where $E, I$ and $k$ are the beam Young's modulus, area moment of inertia and the foundation modulus, respectively. Here $\delta(x)$ is the Dirac delta function. Because a beam can lift-off from a tensionless elastic foundation due to its bending deformation as shown in Fig. 1(a), the beam and tensionless foundation interaction occurs only in the area where the springs are compressed. As a result, the beam deflection $(y)$ is divided into two parts: the contact part of $y_{1}$ and the lift-off part of $y_{2}$. As a tensionless foundation cannot exert tension, the force associated with the foundation vanishes for $y_{2}$. The (unknown) separation points of $x= \pm x_{0}$ demarcate the boundary of the above two governing equations. Here for an infinitely long beam under a transverse concentrated load, the symmetry of the deflection and separation is assumed (Weitsman, 1970). It should be kept in mind that for an infinite beam under complex transverse loads (Ma et al., 2009a), or for a finite beam under an asymmetric transverse concentrated load (Nobili, 2013; Zhang and Murphy, 2004, 2013), or for a beam with the dynamic effects (Chen and Chen, 2011; Kenney, 1954), the above symmetry assumption is not valid. However, as this model is intended for the railway application, it is demonstrated in Appendix A that the beam deflection asymmetry due to the dynamic effects is rather small for the real application in a high- 
a)

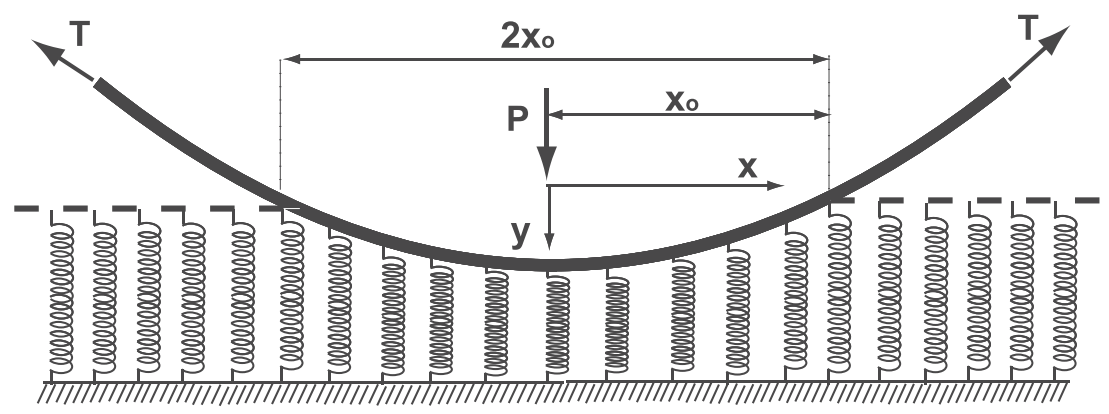

b)

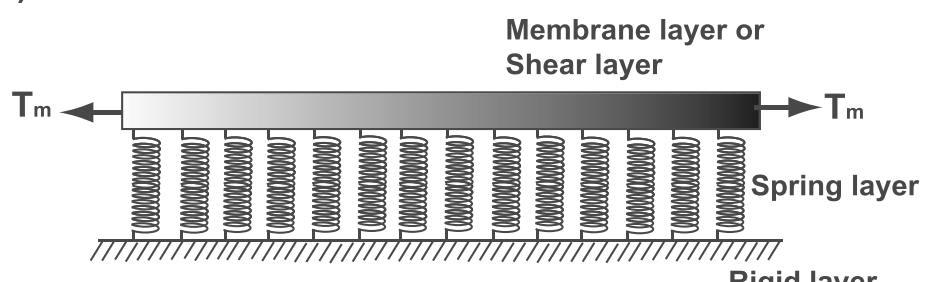

Rigid layer

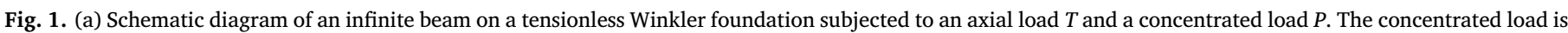

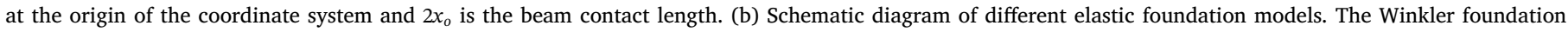

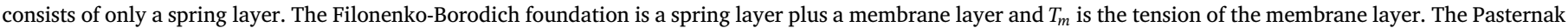
foundation is a spring layer plus a shear layer.

speed railway track (UIC60) (Chen et al., 1997). Furthermore, it is not difficult to extend this symmetric solution of an infinite beam to the finite beam case in which both symmetric and asymmetric contact scenarios can be handled (Zhang and Murphy, 2004).Physically, the Winkler foundation is to idealize an elastic continuum as a layer consisting of closely spaced, identical but mutually independent springs. As a modeling result, the externally applied load is localized in the Winkler foundation, or say, the Winkler foundation only deforms in the loaded area. Therefore, the effect of load dispersion cannot be accounted by the Winkler foundation (Dutta and Roy, 2002; Younesian et al., 2019). Furthermore, the localized deformation behavior of the Winkler foundation causes the deformation discontinuity at the boundary of the loaded area (Dutta and Roy, 2002; Kerr, 1964; Younesian et al., 2019), which in essence violates the compatibility condition of an elastic continuum. To alleviate the demerits of the Winkler foundation model, various elastic foundation models are developed. Besides the spring layer, the Filonenko-Borodich foundation adds a membrane layer and the Pasternak foundation adds a shear layer as shown in Fig. 1(b). The essential improvement of the Filonenko-Borodich foundation and the Pasternak foundation models is that the independent Winkler springs are now connected by the membrane/shear layer and interacts to one another (Dutta and Roy, 2002), which better characterizes an elastic continuum. More importantly, the deformation discontinuity problem is gone (Sapountzakis and Kampitsis, 2011a, 2011b, 2013). The response of the surface of the one dimensional (1D) Filonenko-Borodich foundation subjected to a distributed load $f$ is the following (Dutta and Roy, 2002; Kerr, 1964):

$f=k y-T_{m} \frac{d^{2} y}{d x^{2}}$,

where $T_{m}$ is the membrane tension. For the Pasternak foundation, the following relation holds (Dutta and Roy, 2002; Kerr, 1964): $f=k y-G_{c} \frac{d^{2} y}{d x^{2}}$,

where $G_{c}$ is the shear layer constant. Compared with the FilonenkoBorodich foundation of Eq. (2), $T_{m}$ is replaced by $G_{c}$ in the Pasternak foundation. For a beam resting on the Filonenko-Borodich and Pasternak foundations subjected to a transverse concentrated load, its governing equations are the following two

$E I \frac{d^{4} y}{d x^{4}}-T_{m} \frac{d^{2} y}{d x^{2}}+k y=P \delta(x)$

$E I \frac{d^{4} y}{d x^{4}}-G_{c} \frac{d^{2} y}{d x^{2}}+k y=P \delta(x)$.

The vibration of a beam on the Winkler foundation subjected to a moving transverse concentrated load is described by the following equation (Hetényi, 1946; Timoshenko and Langer, 1932):

$E I \frac{\partial^{4} y}{\partial x_{1}^{4}}+m \frac{\partial^{2} y}{\partial t^{2}}+k y=P \delta\left(x_{1}-v t\right)$,

where $m$ is the beam mass per unit length, $t$ is time and $v$ is the speed of moving load $P$. The above equation describes the dynamic response of a railroad track (modeled as a beam) to a moving concentrated load due to the wheel-track contact (Timoshenko and Langer, 1932) and $x_{1}$ is a fixed coordinate. Its (quasistatic) steady state response is given as the following (Hetényi, 1946; Timoshenko and Langer, 1932):

$E I \frac{d^{4} y}{d x^{4}}+m v^{2} \frac{d^{2} y}{d x^{2}}+k y=P \delta(x)$.

Here $m v^{2}$ is with the unit of Newton and $x=x_{1}-v t$ is a moving coordinate. Eq. (7) states that the track vibration induced by a moving load effectively adds a compressive axial force of $m v^{2}$ (Hetényi, 1946). For the case of constantly moving load, the coordinate system is often set to move with the load for the convenience of study (Kerr, 1972; Lin 
and Adams, 1987).

The railroad deflection causes the rotation of cross-ties and the rotation is resisted by the ballast, which exerts a bending moment on the track. In order to more accurately evaluate the bending stresses of a track, the following equation is given by taking account of the additional bending moment exerted by the ballast (Kerr, 1974).

$E I \frac{d^{4} y}{d x^{4}}-\rho \frac{d^{2} y}{d x^{2}}+k y=P \delta(x)$,

where $\rho$ is a proportionality constant. The mathematical equivalence of Eqs. (4), (5), (7) and (8) to the first equation of Eq. (1) is noticed.The following quantities are introduced

$\xi=\beta x, \quad \xi_{o}=\beta x_{o}, \quad Y_{i}=\beta y_{i}, \alpha=\frac{T \beta^{2}}{k}, F=\frac{P}{4 \beta^{2} E I}, \beta=\sqrt[4]{\frac{k}{4 E I}}$,

where $\alpha$ is the dimensionless axial load and $\beta^{-1}$ is an important characteristic length for a beam on an elastic foundation (Kerr, 1974; Tsai and Westmann, 1967). Eq. (1) is now nondimensionalized as follows

$\left\{\begin{array}{c}\frac{1}{4} Y_{1}^{\prime \prime \prime \prime}-\alpha Y_{1}^{\prime \prime}+Y_{1}=F \delta(\xi),|\xi| \leq \xi_{o}, Y_{1} \geq 0 \\ \frac{1}{4} Y_{2}^{\prime \prime \prime \prime}-\alpha Y_{2}^{\prime \prime}=0,|\xi| \geq \xi_{o}, Y_{2} \leq 0 .\end{array}\right.$

Here ()$^{\prime \prime \prime \prime}=d^{4} / d \xi^{4}$ and ()$^{\prime \prime}=d^{2} / d \xi^{2}$. At the separation points of $\pm \xi_{o}$, the vanishing deflection, moment and shear force result in the following boundary conditions (Weitsman, 1970):

$Y_{1}\left(\xi_{o}\right)=0, Y_{1}^{\prime \prime}\left(\xi_{o}\right)=0, Y_{1}^{\prime \prime \prime}\left(\xi_{o}\right)=0$.

Due to the symmetry, only the three boundary conditions at $\xi=\xi_{o}$ are given and the separation points also define the beam contact length as $2 \xi_{0}$. At $\xi= \pm \xi_{o}$, the two deflections of $Y_{1}$ and $Y_{2}$ are related by the following transversality conditions (Kerr, 1976), which are also variously called the matching conditions (Zhang and Murphy, 2004) or the continuity conditions (Bhattiprolu et al., 2014; 2016)

$Y_{1}\left(\xi_{o}\right)=Y_{2}\left(\xi_{o}\right), Y_{1}^{\prime}\left(\xi_{o}\right)=Y_{2}^{\prime}\left(\xi_{o}\right), Y_{1}^{\prime \prime}\left(\xi_{o}\right)=Y_{2}^{\prime \prime}\left(\xi_{o}\right), Y_{1}^{\prime \prime \prime}\left(\xi_{o}\right)=Y_{2}^{\prime \prime \prime}\left(\xi_{o}\right)$.

Physically, the transversality conditions are to ensure the continuity of the deflection, slope, moment and shear at the separation points (Zhang and Murphy, 2004). Again, because of the symmetry, only the four transversality conditions at $\xi=\xi_{o}$ are given in Eq. (12). In conjunction with the boundary conditions of Eq. (11), the above transversality conditions can also be equivalently written as the following

$Y_{2}\left(\xi_{o}\right)=0, Y_{1}^{\prime}\left(\xi_{o}\right)=Y_{2}^{\prime}\left(\xi_{o}\right), Y_{2}^{\prime \prime}\left(\xi_{o}\right)=0, Y_{2}^{\prime \prime \prime}\left(\xi_{o}\right)=0$.

The $\alpha$ value determines the solution form of Eq. (10) and there are the following five cases in total: no axial load case of $\alpha=0$, three tensile cases of $0<\alpha<1, \alpha=1$ and $\alpha>1$ and one compressive case of $-1<\alpha<0$.

Case I. $\alpha=0$

This is the zero axial load case and the corresponding solution forms of Eq. (10) are the following (Weitsman, 1970):

$\left\{\begin{aligned} Y_{1}= & A_{1} \sinh \xi \sin \xi+B_{1} \cosh \xi \cos \xi+H \sinh |\xi| \cos \xi+G \cosh \xi \sin \\ & |\xi|,|\xi| \leq \xi_{o} \\ Y_{2}= & A_{2} \xi^{3}+B_{2} \xi^{2}+C_{2} \xi+D_{2}, \quad|\xi| \geq \xi_{0} .\end{aligned}\right.$

Here $A_{1}, B_{1}, H, G, A_{2}, B_{2}, C_{2}$ and $D_{2}$ are the eight unknown constants to be determined. Keep in mind that $\xi_{o}$ is also unknown, which is the major reason responsible for the mathematical difficulty of the tensionless contact (Bhattiprolu et al., 2014, 2016; Kerr, 1972; Lin and Adams, 1987; Weitsman, 1970 Zhang and Murphy, 2004). In the above equation, the first two terms of $A_{1} \sinh \xi \sin \xi+B_{1} \cosh \xi \cos \xi$ are the homogeneous solution of $Y_{1}$ and the last two terms of $H \sinh |\xi| \cos \xi+G \cosh \xi \sin |\xi|$ are the particular solution. There are two other homogeneous solution terms associated with the odd functions of $\sinh \xi \cos \xi$ and $\cosh \xi \sin \xi$, which are tossed away. Because the presence of odd functions breaks the deflection symmetry, only even functions survive in the solution form. It is noticed that the particular solution of $H \sinh |\xi| \cos \xi+G \cosh \xi \sin |\xi|$ is the even function constructed from the above two odd functions.

Case II. $0<\alpha<1$

The corresponding solution forms of Eq. (10) are as follows

$\left\{\begin{aligned} Y_{1}= & A_{1} \sinh \left(\alpha_{1} \xi\right) \sin \left(\alpha_{2} \xi\right)+B_{1} \cosh \left(\alpha_{1} \xi\right) \cos \left(\alpha_{2} \xi\right)+H \sinh \left|\alpha_{1} \xi\right| \\ & \cos \left(\alpha_{2} \xi\right)+G \cosh \left(\alpha_{1} \xi\right) \sin \left|\alpha_{2} \xi\right|,|\xi| \leq \xi_{o} \\ Y_{2}= & A_{2} e^{-2 \sqrt{\alpha} \xi}+B_{2} e^{2 \sqrt{\alpha} \xi}+C_{2} \xi+D_{2},|\xi| \geq \xi_{o} .\end{aligned}\right.$

Here $\alpha_{1}$ and $\alpha_{2}$ are defined as $\alpha_{1}=\sqrt{1+\alpha}$ and $\alpha_{2}=\sqrt{1-\alpha}$.

Case III. $\alpha=1$

The corresponding solution forms of Eq. (10) are as follows

$\left\{\begin{aligned} Y_{1}= & A_{1} \xi \sinh (\sqrt{2} \xi)+B_{1} \cosh (\sqrt{2} \xi)+H \sinh |\sqrt{2} \xi|+G|\xi| \cosh (\sqrt{2} \xi) \\ & |\xi| \leq \xi_{o} \\ Y_{2}= & A_{2} e^{-2 \xi}+B_{2} e^{2 \xi}+C_{2} \xi+D_{2},|\xi| \geq \xi_{0} .\end{aligned}\right.$

Case IV. $\alpha>1$

The corresponding solution forms of Eq. (10) are as follows

$\left\{\begin{array}{l}Y_{1}=A_{1} \cosh \left(\gamma_{1} \xi\right)+B_{1} \cosh \left(\gamma_{2} \xi\right)+H \sinh \left|\gamma_{1} \xi\right|+G \sinh \left(\gamma_{2} \xi\right),|\xi| \leq \xi_{o} \\ Y_{2}=A_{2} e^{-2 \xi}+B_{2} e^{2 \xi}+C_{2} \xi+D_{2},|\xi| \geq \xi_{o} .\end{array}\right.$

Here $\gamma_{1}$ and $\gamma_{2}$ are defined as $\gamma_{1}=\sqrt{2 \alpha+2 \sqrt{\alpha^{2}-1}}$ and $\gamma_{2}=\sqrt{2 \alpha-2 \sqrt{\alpha^{2}-1}}$.

Case V. $-1<\alpha<0$

The corresponding solution forms of Eq. (10) are as follows

$\left\{\begin{aligned} Y_{1}= & A_{1} \sinh \left(\alpha_{1}^{*} \xi\right) \sin \left(\alpha_{2}^{*} \xi\right)+B_{1} \cosh \left(\alpha_{1}^{* \xi}\right) \cos \left(\alpha_{2}^{* \xi}\right)+H \sinh \left|\alpha_{1}^{* \xi}\right| \\ & \cos \left(\alpha_{2}^{*} \xi\right)+G \cosh \left(\alpha_{1}^{*} \xi\right) \sin \left|\alpha_{2}^{*} \xi\right|,|\xi| \leq \xi_{o} \\ Y_{2}= & A_{2} \sin (2 \sqrt{\alpha} \xi)+B_{2} \cos (2 \sqrt{\alpha} \xi)+C_{2} \xi+D_{2},|\xi| \geq \xi_{o} .\end{aligned}\right.$

Here $\alpha_{1}^{*}$ and $\alpha_{2}^{*}$ are defined as $\alpha_{1}^{*}=\sqrt{1-|\alpha|}$ and $\alpha_{2}^{*}=\sqrt{1+|\alpha|}$. When $\alpha=-1$, the corresponding axial load $T=-k \beta^{-2}=-2 \sqrt{k E I}$ as defined by Eq. (9) is (compressive) buckling load of an infinite beam on the Winkler foundation that reacts to both tension and compression (Hetényi, 1946). Once the beam buckles and enters the post-buckling region, i.e., $\alpha \leq-1$, the linear equations of Eq. (10) do not apply. This is the reason why we only handle one compression case of $-1<\alpha<0$.

With the above five different solution forms depending on $\alpha$, the separation point of $\xi_{o}$ and eight unknown constants of $A_{1}, B_{1}, H, G, A_{2}$, $B_{2}, C_{2}$ and $D_{2}$ are found in conjunction with the boundary conditions of Eq. (11) and transversality conditions of Eq. (13). The detailed procedures are presented in Appendix B. Because finding the separation point is the major difficulty and a key thing in the tensionless contact problem, the equations for the five cases to determine $\xi_{o}$ are summarized as follows.

Case I. $\alpha=0$

$\cosh \left(\xi_{o}\right) \cos \left(\xi_{o}\right)=0$.

This equation was firstly derived by Weitsman (1970). Clearly, $\xi_{0}=\pi / 2$ is a solution. There are other solutions such as $\xi_{o}=(2 n+1) \pi / 2(n=1,2,3 \ldots)$, which are discarded. The reason is that only $\xi_{o}=\pi / 2$ can ensure $Y_{1} \geq 0$ in the region of $|\xi| \leq \xi_{o}$ (Weitsman, 


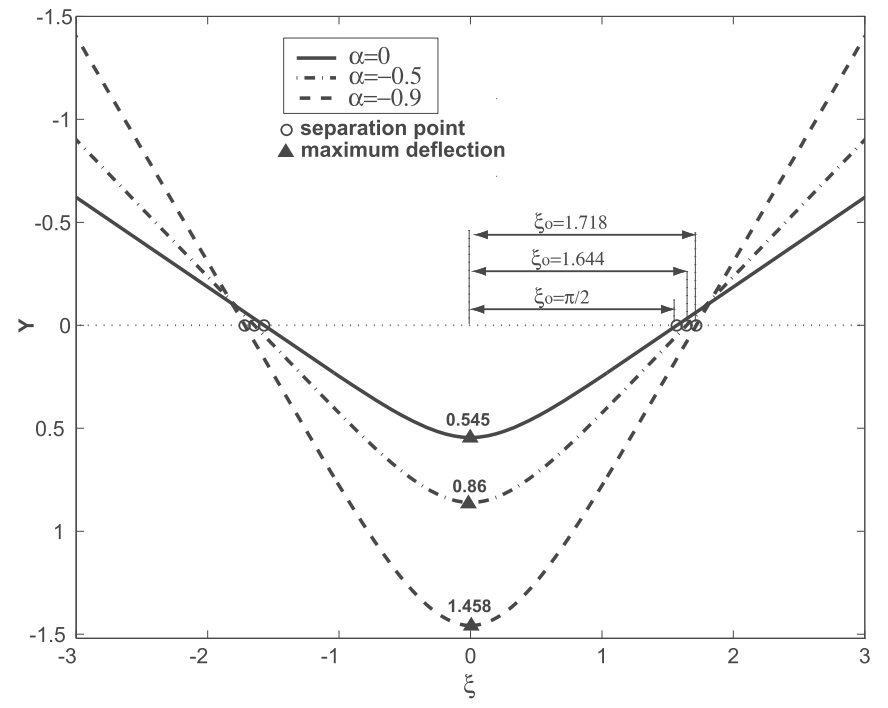

Fig. 2. The beam deflections with three different $\alpha$ s. As the compression increases ( $\alpha$ decreases), the half contact length increases from $\xi_{o}=\pi / 2$ of $\alpha=0$ to $\xi_{o}=1.644$ of $\alpha=-0.5$ and to $\xi_{o}=1.718$ of $\alpha=-0.9$. The beam maximum deflection also increases from $Y(0)=0.545$, to $Y(0)=0.86$ and to $Y(0)=1.458$ for $\alpha=0,-0.5$ and -0.9 , respectively.

\section{0).}

Case II. $0<\alpha<1$

$$
\begin{aligned}
& {\left[2 \alpha \sinh \left(\alpha_{1} \xi_{o}\right) \sin \left(\alpha_{2} \xi_{o}\right)+2 \sqrt{1-\alpha^{2}} \cosh \left(\alpha_{1} \xi_{o}\right) \cos \left(\alpha_{2} \xi_{o}\right)\right]} \\
& \quad\left\{\frac{2\left[\cosh ^{2}\left(\alpha_{1} \xi_{o}\right)+\cos ^{2}\left(\alpha_{2} \xi_{o}\right)\right]}{\operatorname{Det}}+\frac{1}{2}\left(\frac{\alpha_{2}}{\alpha_{1}}+\frac{\alpha_{1}}{\alpha_{2}}\right)\right\} \\
& -\frac{8 \sqrt{1-\alpha^{2}}}{\text { Det }} \cosh \left(\alpha_{1} \xi_{o}\right) \cos \left(\alpha_{2} \xi_{o}\right)=0,
\end{aligned}
$$

where $\quad \alpha_{1}=\sqrt{1+\alpha}, \quad \alpha_{2}=\sqrt{1-\alpha} \quad$ and $\quad$ Det $=-$ $2 \alpha_{1} \alpha_{2}\left[\sinh ^{2}\left(\alpha_{1} \xi_{o}\right) \sin ^{2}\left(\alpha_{2} \xi_{o}\right)+\cosh ^{2}\left(\alpha_{1} \xi_{o}\right) \cos ^{2}\left(\alpha_{2} \xi_{o}\right)\right]$. When $\alpha=0$, $\alpha_{1}=\alpha_{2}=1$ and Eq. (20) becomes the following

$2 \cosh \left(\xi_{o}\right) \cos \left(\xi_{o}\right)\left\{\frac{2\left[\cosh ^{2}\left(\xi_{o}\right)+\cos ^{2}\left(\xi_{o}\right)\right]}{\text { Det }}+1-\frac{4}{\text { Det }}\right\}=0$.

Clearly, Eq. (21) results in $\cosh \left(\xi_{o}\right) \cos \left(\xi_{o}\right)=0$, which recovers Eq. (19) of the $\alpha=0$ case.

\section{Case III. $\alpha=1$}

$-2 \cosh \left(\sqrt{2} \xi_{o}\right)+\sqrt{2} \xi \sinh \left(\sqrt{2} \xi_{o}\right)=0$.

Case IV. $\alpha>1$

$-\gamma_{1}^{3} \frac{\sinh ^{2}\left(\gamma_{1} \xi_{o}\right)}{\cosh \left(\gamma_{1} \xi_{o}\right)}+\gamma_{1} \gamma_{2}^{2} \frac{\sinh ^{2}\left(\gamma_{2} \xi_{o}\right)}{\cosh \left(\gamma_{2} \xi_{o}\right)}+\gamma_{1}^{3} \cosh \left(\gamma_{1} \xi_{o}\right)-\gamma_{1} \gamma_{2}^{2} \cosh \left(\gamma_{2} \xi_{o}\right)=0$,

where $\gamma_{1}=\sqrt{2 \alpha+2 \sqrt{\alpha^{2}-1}}$ and $\gamma_{2}=\sqrt{2 \alpha-2 \sqrt{\alpha^{2}-1}}$.

Case V. $-1<\alpha<0$

$$
\begin{aligned}
& {\left[-2|\alpha| \sinh \left(\alpha_{1}^{*} \xi_{o}\right) \sin \left(\alpha_{2}^{*} \xi_{o}\right)+2 \sqrt{1-\alpha^{2}} \cosh \left(\alpha_{1}^{*} \xi_{o}\right) \cos \left(\alpha_{2}^{*} \xi_{o}\right)\right]} \\
& \left\{\frac{2\left[\cosh ^{2}\left(\alpha_{1}^{*} \xi_{o}\right)+\cos ^{2}\left(\alpha_{2}^{*} \xi_{0}\right)\right]}{D e t^{*}}+\frac{1}{2}\left(\frac{\alpha_{2}^{*}}{\alpha_{1}^{*}}+\frac{\alpha_{1}^{*}}{\alpha_{2}^{*}}\right)\right\} \\
& -\frac{8 \sqrt{1-\alpha^{2}}}{\operatorname{Det}^{*}} \cosh \left(\alpha_{1}^{*} \xi_{o}\right) \cos \left(\alpha_{2}^{* \xi_{o}}\right)=0,
\end{aligned}
$$

where $\quad \alpha_{1}^{*}=\sqrt{1-|\alpha|}, \quad \alpha_{2}^{*}=\sqrt{1+|\alpha|} \quad$ and $\quad D$ $e t^{*}=-2 \alpha_{1}^{*} \alpha_{2}^{*}\left[\sinh ^{2}\left(\alpha_{1}^{*} \xi_{o}\right) \sin ^{2}\left(\alpha_{2}^{*} \xi_{o}\right)+\cosh ^{2}\left(\alpha_{1}^{*} \xi_{o}\right) \cos ^{2}\left(\alpha_{2}^{*} \xi_{o}\right)\right] . \quad$ The resemblance between Eqs. (20) and (24) is noticed. If $\alpha_{1}^{*}$ is replaced by $\alpha_{1}$ and $\alpha_{2}^{*}$ by $\alpha_{2}$, Eq. (24) becomes Eq. (20). Similar to the $0<\alpha<1$ case, when $\alpha=0, \alpha_{1}^{*}=\alpha_{2}^{*}=1$ and Eq. (24) becomes the following

$2 \cosh \left(\xi_{o}\right) \cos \left(\xi_{o}\right)\left\{\frac{2\left[\cosh ^{2}\left(\xi_{o}\right)+\cos ^{2}\left(\xi_{o}\right)\right]}{\text { Det }^{*}}+1-\frac{4}{\text { Det }^{*}}\right\}=0$.

Again, Eq. (25) results in $\cosh \left(\xi_{o}\right) \cos \left(\xi_{o}\right)=0$, which recovers Eq. (19) of the $\alpha=0$ case.

In all the five equations determining $\xi_{o}$, one thing in common is that they do not contain $F$. This is a benchmark characteristics of the tensionless contact of various structures subjected to a single transverse concentrated load (Weitsman, 1970; Zhang and Murphy, 2004). Here the presence of an axial force does not change this benchmark property. A finite element analysis is also carried out to numerically verify this conclusion and ensure the correct derivations of those unknown constants as given in Appendix B. Besides an infinite beam, for a finite beam (Zhang, 2008; Zhang and Murphy, 2004), a finite or infinite plate (Dempsey et al., 1984; Weitsman, 1969; Zhang and Murphy, 2012), the conclusion of the contact length/area independence on a single concentrated load still holds even when the support is modeled as the tensionless Reissner foundation (Zhang, 2008) or as the tensionless elastic half-space (Weitsman, 1972).

\section{Results and discussion}

Depending on the $\alpha$ value, the separation point of $\xi_{o}$ is determined by Eq. (19)-(23). Except Eq. (19), the other four equations have to be numerically solved by the Newton-Rhapson method (Press et al., 1986). In Eq. (19)-(23), the concentrated load of $F$ has no impact on $\xi_{0}$. As seen from the solution forms of the eight unknown constants presented in Appendix B, $F$ acts as a proportionality factor which modulates the magnitude of the beam deflection. In all the results presented in this study, $F=1$ is taken.

Fig. 2 plots the beam deflections with $\alpha=0,-0.5$ and -0.9 , respectively. The deflection with $\alpha=0$ is described by Eq. (14) and the other two are described by Eq. (18). The separation points are marked by circles. As the axial compression increases, i.e., the decreasing negative $\alpha$, both the contact length and the deflection magnitude enlarge: $\xi_{o}=\pi / 2,1.644,1.718$ and $Y(0)=0.545,0.86,1.458$ for $\alpha=0,-0.5$ and -0.9 , respectively. Because the compressive axial load reduces the system effective stiffness, the beam has to increase its contact length and deflection to balance the concentrated load. In conjunction with Eq. (18) and Eq. (41) in Appendix B, we have $\lim _{\alpha \rightarrow-1} Y_{1}(0)=B_{1} \rightarrow \infty$, which is the buckling definition of the system effective stiffness becoming zero (Hetényi, 1946). Therefore, the buckling loads of an infinite beam on the Winkler foundation and the tensionless Winkler foundation are the same as $\alpha=-1$. The reason is that upon the buckling, there is no beam (upward) deflection and the effects of the two Winkler foundations are thus the same. However, once the beam buckles and enters the post-buckling region with an upward beam deflection, there is an instant difference between these two foundations (Kerr and El-Aini, 1978). It is also interesting to notice that though the deflections of a beam on these two foundations become infinite upon the buckling load, their contact length and wavelength are finite. The computation of Eq. (24) yields $\lim _{\alpha \rightarrow-1} \xi_{o} \approx 1.738$. In comparison, the half wavelength of the buckling beam on the Winkler foundation that reacts to both tension and compression is $\Lambda=\pi / \sqrt{2 E I /|T|}$ (Hetényi, 1946) and its corresponding dimensionless form is $\lambda=\beta \Lambda=\pi / \sqrt{2} \approx 2.22$. Fig. 3 shows the beam deflections with $\alpha=0.5$, 1 and 1.5, respectively. The deflections are described by Eq. (16) for $\alpha=1$ and by Eq. (15) for $\alpha=0.5$ and $\alpha=1.5$. As the tension ( $\alpha$ ) increases, both the contact length and deflection magnitude decrease: $\xi_{o}=1.511,1.46,1.417$ and $Y(0)=0.389,0.297,0.237$ for $\alpha=0.5,1$ and 1.5 , respectively. The reason is that the tensile axial load increases the system effective stiffness and as a result, smaller contact length and deflection are needed to balance the concentrated load. In Eq. (1) or 


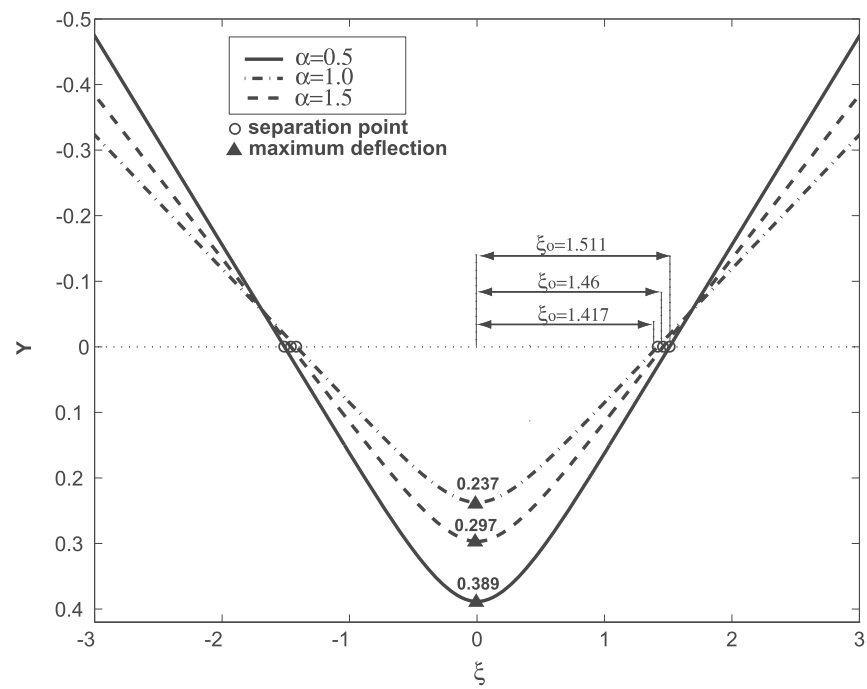

Fig. 3. The beam deflections under tensile axial loads with three different positive $\alpha$ s. As the tension increases, the half contact length now decreases from $\xi_{o}=1.511$ of $\alpha=0.5$, to $\xi_{o}=1.46$ of $\alpha=1$ and to $\xi_{o}=1.417$ of $\alpha=1.5$. The beam maximum deflection also decreases from $Y(0)=0.389$, to $Y(0)=0.297$ and to $Y(0)=0.237$ for $\alpha=0.5,1$ and 1.5 , respectively.

(10), an implicit assumption is that there is only one contact zone of $|x| \leq x_{o}$ (Weitsman, 1970). This assumption is valid for the tensionless foundation. The foundation of a ballastless high-speed railway demonstrates some (weak) capability of withholding tension, which is described an asymmetric bilinear foundation model (Zhang et al., 2018). In the bilinear foundation model, there are multiple contact zones for an infinite beam/track under a concentrated load (Zhang et al., 2018). However, in the contact zone where the concentrated load is located, there are little differences of the beam deflections and contact stresses as predicated by the tensionless foundation and the bilinear foundation models (Zhang et al., 2018). The mechanism for this little difference is that the major deformations of both the elastic foundation and beam are in a small (contact) region around the concentrated load (Zhang et al., 2018).

We need to address an important issue on the beam deflection in the lift-off zone. For all five cases, $A_{2}=B_{2}=0$ in the lift-off zone of $\xi>\xi_{0}$. Therefore, all the beam deflections with different $\alpha$ values are described by $Y_{2}(\xi)=C_{2} \xi+D_{2}$. Clearly, $Y_{2}(\xi)$ is a straight line and it approaches infinity as $\xi$ approaches infinity, which is unphysical. However, this deflection configuration does satisfy the equilibrium equation of Eq. (10). Because $Y_{2}^{\prime \prime}(\xi)=Y_{2}^{\prime \prime \prime}(\xi) \equiv 0$, the $\xi>\xi_{o}$ part of the beam deflection has no contribution to the moment and shear, which is the reason why it can go to infinity without violating the equilibrium equation. As mentioned above, this unphysical feature of the beam deflection going to infinity can be easily overcome by considering the distributed load of the beam self-weight (Tsai and Westmann, 1967; Weitsman, 1970, 1972). While, for the tensionless contact under a concentrated load, the focus is on the contact zone (Weitsman, 1970, 1972), in which the deflection results are reliable.

The beam bending moment, which is given as $M=-E I y^{\prime \prime}=-E I \beta Y^{\prime \prime}$, determines the beam maximum axial stress as $\sigma_{\max }=-M y_{b} / I$. Here $y_{b}$ is the distance between the beam cross-section surface(s) and its neutral axis. For a given beam, $y_{b}$ is fixed. The maximum axial stress, which is directly associated with the curvature of $Y^{\prime \prime}$, is an important parameter of evaluating the stress, fatigue and fracture of a railroad track (Hetényi, 1946; Timoshenko and Langer, 1932). Figs. 4 and 5 present the beam curvatures under different axial load. In Fig. 4, the curvatures of $\alpha=0,-0.5$ and -0.9 are presented. Clearly, the maximum value of $\left|Y^{\prime \prime}(\xi)\right|$ is at $\xi=0$ and it monotonically decreases to zero as $\xi$ increases. For $\alpha=0,-0.5$ and -0.9 , the maximum curvature values are $\left|Y^{\prime \prime}(0)\right|=1.09,\left|Y^{\prime \prime}(0)\right|=1.441$ and $\left|Y^{\prime \prime}(0)\right|=2.048$,

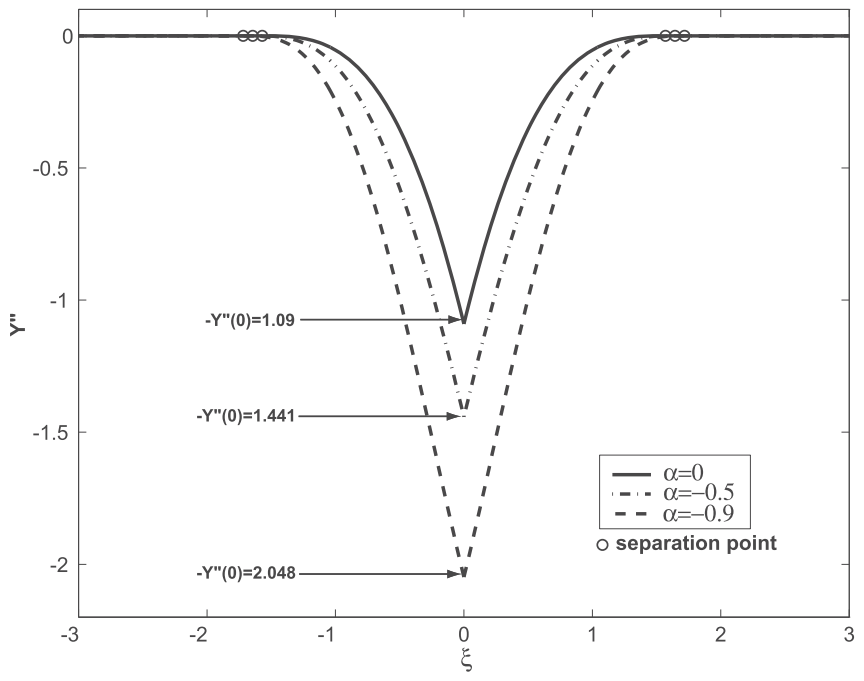

Fig. 4. The curvatures of $\alpha=0,-0.5$ and -0.9 . The separation points marked by circles are the same as those in Fig. 2 .

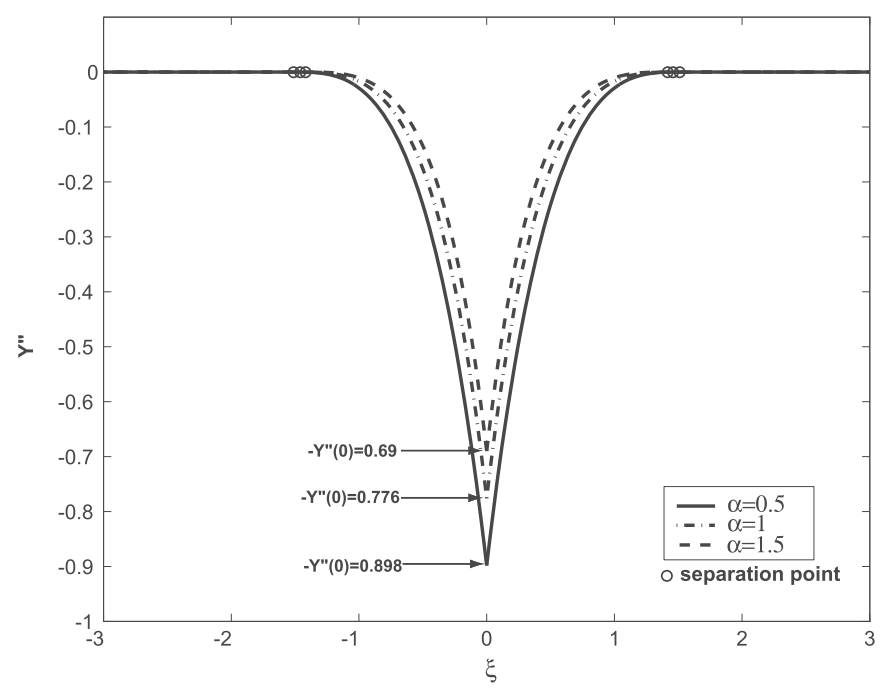

Fig. 5. The curvatures of $\alpha=0.5,1$ and 1.5. The separation points marked by circles are the same as those in Fig. 3.

respectively. As also seen in Fig. 4, a larger compression results in a larger $\left|Y^{\prime \prime}(\xi)\right|$ at any given point of $\xi$. For $\alpha=0.5,1$ and 1.5 in Fig. 5, $\left|Y^{\prime \prime}(0)\right|=0.898, \quad\left|Y^{\prime \prime}(0)\right|=0.776$ and $\left|Y^{\prime \prime}(0)\right|=0.69$, respectively. Clearly, a larger tension results in a smaller $\left|Y^{\prime \prime}(\xi)\right|$ at any given point of $\xi$. As seen in Fig. 2, the beam under a larger axial compression is with a larger contact length but a significantly larger deflection magnitude. Therefore, the variations of both the beam deflection and slope are much larger in a slightly larger contact area, which results in a larger curvature and thus a larger bending stress. The similar argument also explains why a larger tension results in a smaller curvature. In summary, the presence of compressive axial load increases the bending stress and thus makes the stress concentration at the concentrated load locus more severe, which may significantly reduce the life span of a track. On the other hand, the presence of tensile axial load mitigates the stress concentration problem by distributing the bending stress more uniformly. In the track-wheel contact application, a larger train speed results in a larger axial compression as reflected by Eq. (7), which is harmful to a railroad track. In conjunction with Eqs. (4) and (5), besides exerting axial tension on the track, increasing the tension of membrane layer $\left(T_{m}\right)$ or the shear layer constant $\left(G_{c}\right)$ is also an effective method of reducing the stress concentration and improving the system stiffness. A reinforced bed overlaying a soft soil stratum is often used to improve 
a)
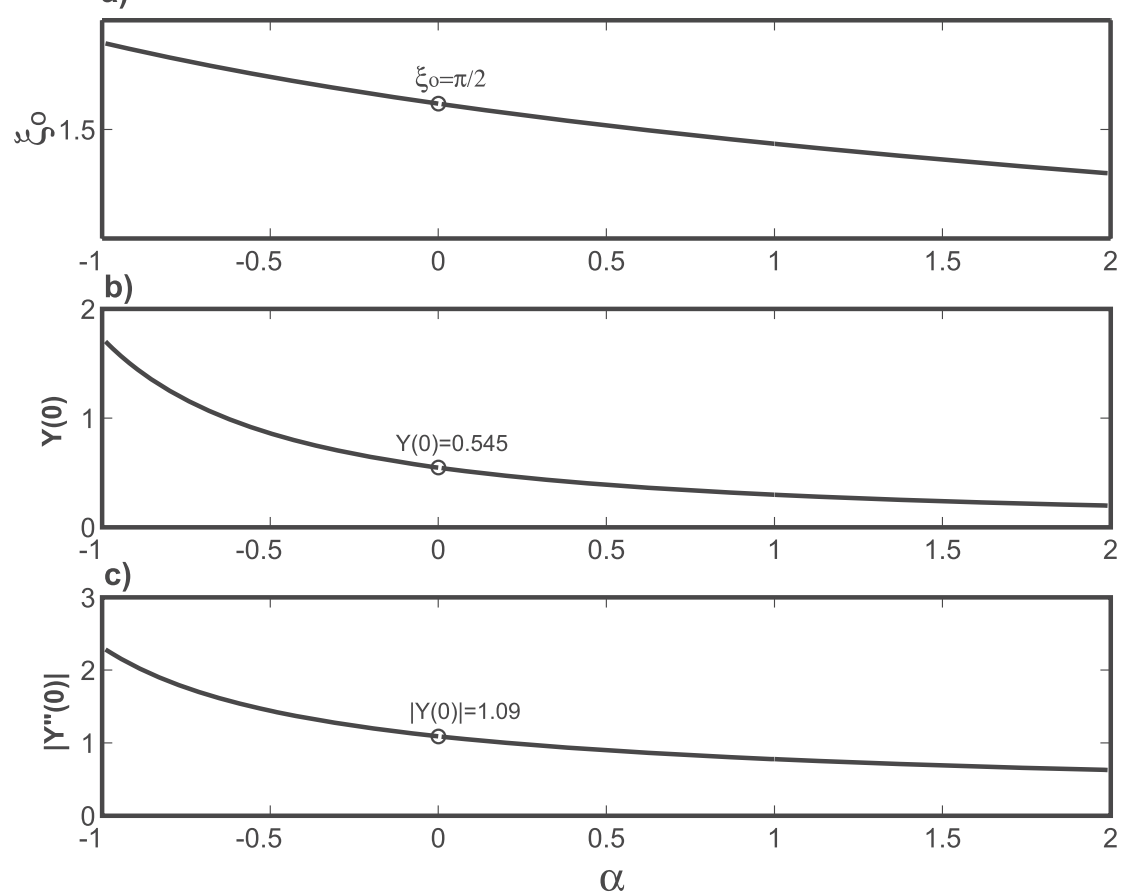

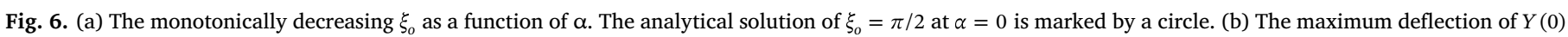

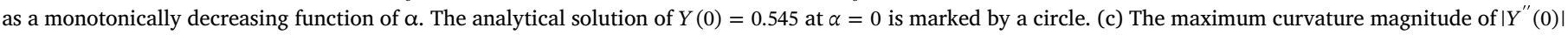
as a monotonically decreasing function of $\alpha$. The analytical solution of $\left|Y^{\prime \prime}(0)\right|=1.09$ at $\alpha=0$ is marked by a circle.

the bearing capacity of a rail-foundation system. The reinforced bed consists of two layers: the granular fill layer and the geosynthetic layer (Maheshwari et al., 2004). In the viewpoint of modeling, the granular fill layer is the shear layer of the Pasternak foundation and the geosynthetic layer is the membrane layer of the Filonenko-Borodich foundation (Maheshwari et al., 2004).

In Fig. 6, the above results are more succinctly summarized. Fig. 6 (a), (b) and (c) present the half contact length $\left(\xi_{0}\right)$, beam deflection magnitude $(Y(0))$ and curvature $\left(\left|Y^{\prime \prime}(0)\right|\right)$ as the functions of the axial load $(\alpha)$. These three quantities are all monotonically decreasing and nonlinear functions of $\alpha$. The special case of $\alpha=0$ was analytically solved by Weitsman (1970) and the related results are marked with circles in Fig. 6. As discussed above, for all different $\alpha$, $Y(\xi) \propto F$ and therefore, both the displacement and curvature at any given point of $\xi$ will vary linearly with $F$. Fig. 7 plots the force-displacement of $F-Y(0)$ with different $\alpha$ s. The linear relation between $F$ and $Y(\xi)$ also implies that for different transverse loads of $F$, only one $F-Y(\xi)$ data is needed and all others can be easily extracted by this linearity relation.

\section{Conclusion}

Depending on the value of the axial load, the solution form of a beam on the tensionless Winkler foundation varies. Total five different solution forms are studied and the corresponding five equations of determining the separation point are derived. Once the separation point is numerically solved, the closed form expressions of the beam deflection can be found. Under a same transverse concentrated load, the compressive axial load reduces the system stiffness and thus enlarges the beam deflection, which also leads to a larger bending stress concentration. On the other hand, the tensile axial load increases the system stiffness, which results in both smaller beam deflection and smaller bending stress. The tensile axial load can thus be utilized as an effective method to relieve the stress concentration. For the tensionless contact of an infinite beam subjected to a transverse concentrated load, a hallmark property is that the concentrated load has no influence on the contact length whatever the axial load is. The effect of a concentrated load is to linearly determine the magnitude of the

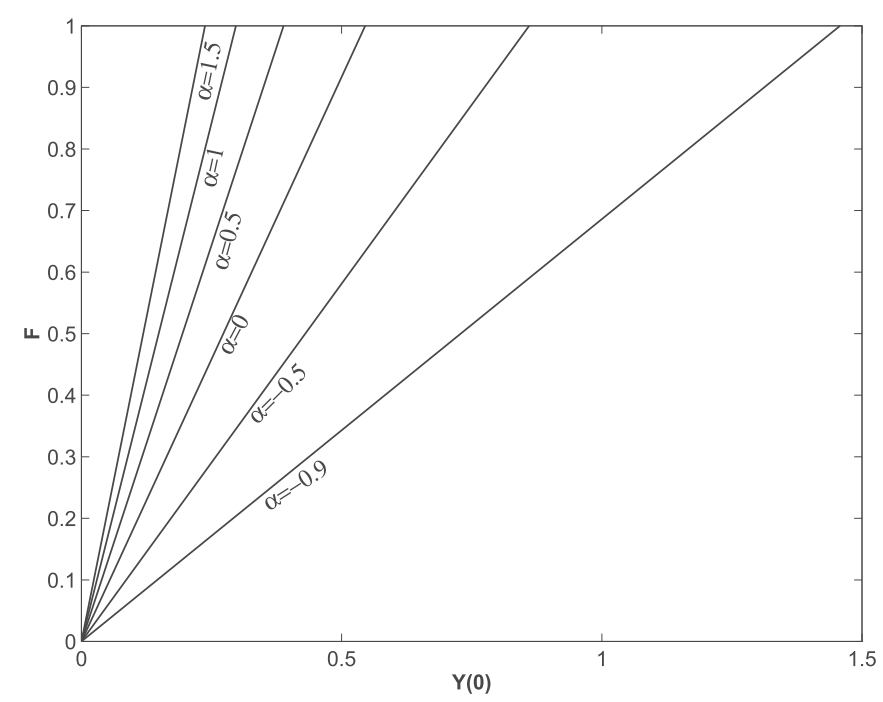

Fig. 7. The linear force-displacement relations of $F-Y(0)$ with different as.

beam deflection. This outstanding property means that for the response of an infinite beam on a tensionless foundation subjected to a given axial load and varying transverse concentrated loads, only one computation in essence is needed and all other results can then be easily obtained by multiplying a numerical factor.

\section{Acknowledgments}

This work was supported by the National Key Research and Development Program of China (2017YFA0204402, 2016YFB1200602-09 and 2016YFB1200602-10), the National Natural Science Foundation of China (NSFC Nos. 11772335 and 11772334), the Strategic Program Research Program (B) of the Chinese Academy of Sciences (XDB22020201). 


\section{Appendix A. The Beam asymmetric deflections under a moving concentrated with the presence of damping}

With the presence of damping, Eq. (7) of our manuscript becomes the following by adding a damping term (Kenney, 1954)

$E I \frac{\partial^{4} y}{\partial x^{4}}+m \frac{\partial^{2} y}{\partial t^{2}}+c \frac{\partial y}{\partial t}+k y=P \delta(x-v t)$,

where $v$ is the load $P$ moving speed. The following quantities are introduced (Kenney, 1954):

$\lambda=\sqrt[4]{\frac{k}{4 E I}}, v_{c r}=\sqrt[4]{\frac{4 k E I}{m^{2}}}, c_{r}=2 \sqrt{k m}, Y=\lambda y, \xi=\lambda(x-v t), \theta=\frac{v}{v_{c r}}, \beta=\frac{c}{c_{r}}, F=\frac{4 P \lambda^{2}}{k}$,

where $v_{c r}$ and $c_{r}$ are the critical speed and damping, respectively. Here $\xi=\lambda(x-v t)$ is a moving coordinate. The dimensionless steady-state of Eq. (26) in a moving coordinate system with a constant speed is written as follows:

$\frac{\partial^{4} Y}{\partial \xi^{4}}+4 \theta^{2} \frac{\partial^{2} Y}{\partial \xi^{2}}-8 \theta \beta \frac{\partial Y}{\partial \xi}+4 Y=F \delta(\xi)$.

Clearly, $4 \theta^{2} \partial^{2} Y / \partial \xi^{2}$ is an effective axial compression induced by the moving concentrated load. Due to the presence of damping, the derivation of the (approximate) analytical solution is complex and very lengthy (Kenney, 1954). Here we only present the results based on the Kenney's (1954) solution to Eq. (28).

Fig. A1 plots the wave shapes of $\theta=1$ and $\theta=2$ with the underdamping of $\beta=0.1$, which is also Fig. 1(b) presented by Kenney (1954). Here $\theta=1$ is the (dimensionless) critical moving speed. With the presence of damping, $\theta=1$ and $\theta=2$ are the subcritical and supercritical cases, respectively. The symmetric static solution (Hetényi, 1946) is also plotted for a comparison. Clearly, the asymmetric wave shapes and multiple contact zones arise in both cases with the presence of damping. Besides damping, the moving speed also determines the asymmetry. Even with no damping $(\beta=0)$, the asymmetry of wave shape can still arise when a moving speed surpasses the critical speed (Kenney, 1954). This causes an even more outstanding asymmetry for the supercritical case as seen in Fig. A1. With such high moving speeds of $\theta=1$ and $\theta=2$, the assumptions of symmetry and one contact zone are indeed invalid.

Fig. A2 plots the wave shapes of $\theta=0.1$ and $\theta=0.2$ in comparison with the static deflection. The same $\beta=0.1$ is taken. In the subcritical range of moving speed, it is actually the combined effect of moving speed and damping which determines the asymmetry. As seen in Fig. A2, there is little difference between these three curves. Because the static one is symmetric and with only one contact zone, the symmetry and one contact zone assumptions are good ones for $\theta=0.1$ and $\theta=0.2$. Furthermore, the "small" speeds of $\theta=0.1$ and $\theta=0.2$ may physically correspond to very large "absolute" speeds. For a high-speed railway track (UIC60) (Chen et al., 1997), its parameters are the following: $E=2 \times 10^{11} \mathrm{~N} / \mathrm{m}^{2}, I=3.06 \times 10^{-5}$ $\mathrm{m}^{4}$ and $m=60.34 \mathrm{~kg} / \mathrm{m}$. The foundation modulus varies in a large range of $5 \times 10^{6} \mathrm{~N} / \mathrm{m}^{2} \leq k \leq 10^{9} \mathrm{~N} / \mathrm{m}^{2}$ (Chen and Chen, 2011) and here a very moderate value of $k=1.67 \times 10^{7} \mathrm{~N} / \mathrm{m}^{2}$ is taken. The corresponding critical speed and damping defined in Eq. (27) are with the following fixed values: $v_{c r}=572.7 \mathrm{~m} / \mathrm{s}\left(2061.7 \mathrm{~km}\right.$ per hour) and $c_{r}=62134 \mathrm{kgm}^{-1} \mathrm{~s}^{-1}$. As a result, $\theta=0.1$ and $\theta=0.2$ in Fig. A2 physically correspond to the train speed of $206.17 \mathrm{~km}$ per hour and $412.34 \mathrm{~km}$ per hour, respectively. Currently, there are no operating trains with a speed higher than $400 \mathrm{~km}$ per hour.

Here a noteworthy point is that the governing equation of Eq. (10) in conjunction with the transversality condition of Eq. (12) is capable of handling the asymmetric contact scenario (Zhang and Murphy, 2004). The symmetry assumption simply reduces the mathematical difficulty of derivation.

\section{Appendix B. Determining the unknowns of the closed form solution}

To determine the nine unknowns of $\xi_{o}, A_{1}, B_{1}, H, G, A_{2}, B_{2}, C_{2}$ and $D_{2}$ in Eqs. (14)-(18), the following three steps are carried out: The first is to find $H$ and $G$; the second is to find $\xi_{o}$ and the third is to find $A_{1}, B_{1}, A_{2}, B_{2}, C_{2}$ and $D_{2}$. This three-step procedure applies to all five cases of different $\alpha$ values. Because $\alpha=0$ and $\alpha=1$ are the two special cases and $0<\alpha<1, \alpha>1$ and $-1<\alpha<0$ are the three general cases, here we demonstrates the solution procedure for the $0<\alpha<1$ case.

The solution form of the $0<\alpha<1$ case, the particular solution of $Y_{1}$ as given by Eq. (15) is $H \sinh \left|\alpha_{1} \xi\right| \cos \left(\alpha_{2} \xi\right)+G \cosh \left(\alpha_{1} \xi\right) \sin \left|\alpha_{2} \xi\right|=H f_{1}+G f_{2}$. By definition, $f_{1}=\sinh \left|\alpha_{1} \xi\right| \cos \left(\alpha_{2} \xi\right)$ and $f_{2}=\cosh \left(\alpha_{1} \xi\right) \sin \left|\alpha_{2} \xi\right|$. The successive differentiations on $f_{1}$ yield the following

$f_{1}^{\prime}=\alpha_{1} \cosh \left(\alpha_{1} \xi\right) \operatorname{sgn}(\xi) \cos \left(\alpha_{2} \xi\right)-\alpha_{2} \sinh \left|\alpha_{1} \xi\right| \sin \left(\alpha_{2} \xi\right)$,

$f_{1}^{\prime \prime}=\left(\alpha_{1}^{2}-\alpha_{2}^{2}\right) \sinh \left|\alpha_{1} \xi\right| \cos \left(\alpha_{2} \xi\right)-2 \alpha_{1} \alpha_{2} \cosh \left(\alpha_{1} \xi\right) \sin \left|\alpha_{2} \xi\right|+2 \alpha_{1} \delta(\xi)$,

${f_{1}^{\prime \prime}}^{\prime \prime}=\left(\alpha_{1}^{2}-\alpha_{2}^{2}\right)\left[\alpha_{1} \cosh \left(\alpha_{1} \xi\right) \operatorname{sgn}(\xi) \cos \left(\alpha_{2} \xi\right)-\alpha_{2} \sinh \left|\alpha_{1} \xi\right| \sin \left(\alpha_{2} \xi\right)\right]$

$-2 \alpha_{1} \alpha_{2}\left[\alpha_{1} \sinh \left(\alpha_{1} \xi\right) \sin \left|\alpha_{2} \xi\right|+\alpha_{2} \cosh \left(\alpha_{1} \xi\right) \cos \left(\alpha_{2} \xi\right) \operatorname{sgn}(\xi)\right]+2 \alpha_{1} \delta^{\prime}(\xi)$,

${f_{1}^{\prime \prime \prime}}^{\prime \prime \prime}=\left(\alpha_{1}^{2}-\alpha_{2}^{2}\right)\left[\left(\alpha_{1}^{2}-\alpha_{2}^{2}\right) \sinh \left|\alpha_{1} \xi\right| \cos \left(\alpha_{2} \xi\right)-2 \alpha_{1} \alpha_{2} \cosh \left(\alpha_{1} \xi\right) \sin \left|\alpha_{2} \xi\right|\right]$

$-2 \alpha_{1} \alpha_{2}\left[\left(\alpha_{1}^{2}-\alpha_{2}^{2}\right) \cosh \left(\alpha_{1} \xi\right) \sin \left|\alpha_{2} \xi\right|+2 \alpha_{1} \alpha_{2} \sinh \left|\alpha_{1} \xi\right| \cos \left(\alpha_{2} \xi\right)\right]$

$+\left[2 \alpha_{1}\left(\alpha_{1}^{2}-\alpha_{2}^{2}\right)-4 \alpha_{1} \alpha_{2}^{2}\right] \delta(\xi)+2 \alpha_{1} \delta^{\prime \prime}(\xi)$,

where $\operatorname{sgn}(\xi)$ is the sign function, $\alpha_{1}=\sqrt{1+\alpha}$ and $\alpha_{2}=\sqrt{1-\alpha}$. In a similar way, we can obtain the second and fourth derivatives of $f_{2}$ as follows

$$
\begin{aligned}
f_{2}^{\prime \prime}= & \left(\alpha_{1}^{2}-\alpha_{2}^{2}\right) \cosh \left(\alpha_{1} \xi\right) \sin \left|\alpha_{2} \xi\right|+2 \alpha_{1} \alpha_{2} \sinh \left|\alpha_{1} \xi\right| \cos \left(\alpha_{2} \xi\right)+2 \alpha_{2} \delta(\xi), \\
f_{2}^{\prime \prime \prime}= & \left(\alpha_{1}^{2}-\alpha_{2}^{2}\right)\left[\left(\alpha_{1}^{2}-\alpha_{2}^{2}\right) \cosh \left(\alpha_{1} \xi\right) \sin \left|\alpha_{2} \xi\right|+2 \alpha_{1} \alpha_{2} \sinh \left|\alpha_{1} \xi\right| \cos \left(\alpha_{2} \xi\right)\right] \\
& +2 \alpha_{1} \alpha_{2}\left[\left(\alpha_{1}^{2}-\alpha_{2}^{2}\right) \sinh \left|\alpha_{1} \xi\right| \cos \left(\alpha_{2} \xi\right)-2 \alpha_{1} \alpha_{2} \cosh \left(\alpha_{1} \xi\right) \sin \left|\alpha_{2} \xi\right|\right] \\
& +\left[2 \alpha_{2}\left(\alpha_{1}^{2}-\alpha_{2}^{2}\right)-4 \alpha_{1}^{2} \alpha_{2}\right] \delta(\xi)+2 \alpha_{2} \delta^{\prime}(\xi) .
\end{aligned}
$$

By substituting the particular solution and its derivatives of $H f_{1}+G f_{2}, H f_{1}^{\prime \prime}+G f_{2}^{\prime \prime}$ and $H f_{1}^{\prime \prime \prime \prime}+G f_{2}^{\prime \prime \prime \prime}$ into the first equation of Eq. (10), only the $\delta(\xi)$ and $\delta^{\prime \prime}(\xi)$ terms survive. Equating the coefficients of $\delta(\xi)$ and $\delta^{\prime \prime}(\xi)$, we have the following two equations after some simple manipulations using the relations of $\alpha_{1}^{2}-\alpha_{2}^{2}=2 \alpha$ and $\alpha_{1} \alpha_{2}=\sqrt{1-\alpha^{2}}$ : 
$\left\{\begin{array}{l}\left(-\alpha \alpha_{1}-\sqrt{1-\alpha^{2}} \alpha_{2}\right) H+\left(-\alpha \alpha_{2}+\sqrt{1-\alpha^{2}} \alpha_{1}\right) G=F, \\ \alpha_{1} H+\alpha_{2} G=0 .\end{array}\right.$

Now $H$ and $G$ are solved as the following:

$H=-\frac{F}{2 \alpha_{1}}, G=\frac{F}{2 \alpha_{2}}$.

Substituting the above $H$ and $G$ into $Y_{1}$ of Eq. (15) and taking the derivative successively, we have the following relations at $\xi=\xi_{o}$

$$
\begin{gathered}
Y_{1}\left(\xi_{o}\right)=A_{1} \sinh \left(\alpha_{1} \xi_{o}\right) \sin \left(\alpha_{2} \xi_{o}\right)+B_{1} \cosh \left(\alpha_{1} \xi_{o}\right) \cos \left(\alpha_{2} \xi_{o}\right)-\frac{F}{2 \alpha_{1}} \sinh \left(\alpha_{1} \xi_{o}\right) \cos \left(\alpha_{2} \xi_{o}\right) \\
+\frac{F}{2 \alpha_{2}} \cosh \left(\alpha_{1} \xi_{o}\right) \sin \left(\alpha_{2} \xi_{o}\right), \\
\quad+\frac{F}{2}\left(\frac{\alpha_{2}}{\alpha_{1}}+\frac{\alpha_{1}}{\alpha_{2}}\right) \sinh \left(\alpha_{1} \xi_{o}\right) \sin \left(\alpha_{2} \xi_{o}\right), \\
Y_{1}^{\prime}\left(\xi_{o}\right)=\left(A_{1} \alpha_{2}+B_{1} \alpha_{1}\right) \sinh \left(\alpha_{1} \xi_{o}\right) \cos \left(\alpha_{2} \xi_{o}\right)+\left(A_{1} \alpha_{1}-B_{1} \alpha_{2}\right) \cosh \left(\alpha_{1} \xi_{o}\right) \sin \left(\alpha_{2} \xi_{o}\right) \\
Y_{1}^{\prime}\left(\xi_{o}\right)=\left[A_{1}\left(\alpha_{1}^{2}-\alpha_{2}^{2}\right)-2 B_{1} \alpha_{1} \alpha_{2}\right] \sinh \left(\alpha_{1} \xi_{o}\right) \sin \left(\alpha_{2} \xi_{o}\right)+\left[B_{1}\left(\alpha_{1}^{2}-\alpha_{2}^{2}\right)+2 A_{1} \alpha_{1} \alpha_{2}\right] \cosh \left(\alpha_{1} \xi_{o}\right) \cos \left(\alpha_{2} \xi_{o}\right) \\
+\frac{F}{2}\left(\frac{\alpha_{2}}{\alpha_{1}}+\frac{\alpha_{1}}{\alpha_{2}}\right)\left[\alpha_{2} \sinh \left(\alpha_{1} \xi_{o}\right) \cos \left(\alpha_{2} \xi_{o}\right)+\alpha_{1} \cosh \left(\alpha_{1} \xi_{o}\right) \sin \left(\alpha_{2} \xi_{o}\right)\right], \\
Y_{1}^{\prime \prime}\left(\xi_{o}\right)=\left[A_{1} \alpha_{2}\left(3 \alpha_{1}^{2}-\alpha_{2}^{2}\right)+2 B_{1} \alpha_{1}\left(\alpha_{1}^{2}-3 \alpha_{2}^{2}\right)\right] \sinh \left(\alpha_{1} \xi_{o}\right) \cos \left(\alpha_{2} \xi_{o}\right) \\
+\left[A_{1} \alpha_{1}\left(\alpha_{1}^{2}-3 \alpha_{2}^{2}\right)+2 B_{1} \alpha_{2}\left(\alpha_{2}^{2}-3 \alpha_{1}^{2}\right)\right] \cosh \left(\alpha_{1} \xi_{o}\right) \sin \left(\alpha_{2} \xi_{o}\right) \\
+\frac{F}{2}\left(\frac{\alpha_{2}}{\alpha_{1}}+\frac{\alpha_{1}}{\alpha_{2}}\right)\left[\left(\alpha_{1}^{2}-\alpha_{2}^{2}\right) \sinh \left(\alpha_{1} \xi_{o}\right) \sin \left(\alpha_{2} \xi_{o}\right)+2 \alpha_{1} \alpha_{2} \cosh \left(\alpha_{1} \xi_{o}\right) \cos \left(\alpha_{2} \xi_{o}\right)\right] .
\end{gathered}
$$

Applying the first two boundary conditions of $Y_{1}\left(\xi_{0}\right)=0$ and $Y_{1}^{\prime \prime}\left(\xi_{o}\right)=0$ as given in Eq. (11), we obtain the following two equations after some simple manipulations

$\left\{\begin{array}{l}\sinh \left(\alpha_{1} \xi_{o}\right) \sin \left(\alpha_{2} \xi_{o}\right) A_{1}+\cosh \left(\alpha_{1} \xi_{o}\right) \cos \left(\alpha_{2} \xi_{o}\right) B_{1}=\frac{F}{2 \alpha_{1}} \sinh \left(\alpha_{1} \xi_{o}\right) \cos \left(\alpha_{2} \xi_{o}\right)-\frac{F}{2 \alpha_{2}} \cosh \left(\alpha_{1} \xi_{o}\right) \sin \left(\alpha_{2} \xi_{o}\right), \\ {\left[\left(\alpha_{1}^{2}-\alpha_{2}^{2}\right) \sinh \left(\alpha_{1} \xi_{o}\right) \sin \left(\alpha_{2} \xi_{o}\right)+2 \alpha_{1} \alpha_{2} \cosh \left(\alpha_{1} \xi_{o}\right) \cos \left(\alpha_{2} \xi_{o}\right)\right] A_{1}} \\ +\left[\left(\alpha_{1}^{2}-\alpha_{2}^{2}\right) \cosh \left(\alpha_{1} \xi_{o}\right) \cos \left(\alpha_{2} \xi_{o}\right)-2 \alpha_{1} \alpha_{2} \sinh \left(\alpha_{1} \xi_{o}\right) \sin \left(\alpha_{2} \xi_{o}\right)\right] B_{1} \\ =-\frac{F}{2}\left(\frac{\alpha_{2}}{\alpha_{1}}+\frac{\alpha_{1}}{\alpha_{2}}\right)\left[\alpha_{2} \sinh \left(\alpha_{1} \xi_{o}\right) \cos \left(\alpha_{2} \xi_{o}\right)+\alpha_{1} \cosh \left(\alpha_{1} \xi_{o}\right) \sin \left(\alpha_{2} \xi_{o}\right)\right]\end{array}\right.$

Now $A_{1}$ and $B_{1}$ are solved as the following:

$A_{1}=\frac{F\left[\alpha_{1} \sinh \left(\alpha_{1} \xi_{0}\right) \cosh \left(\alpha_{1} \xi_{0}\right)+\alpha_{2} \sin \left(\alpha_{2} \xi_{0}\right) \cos \left(\alpha_{2} \xi_{0}\right)\right]}{D e t}$,

$B_{1}=\frac{F\left[-\alpha_{2} \sinh \left(\alpha_{1} \xi_{0}\right) \cosh \left(\alpha_{1} \xi_{0}\right)+\alpha_{1} \sin \left(\alpha_{2} \xi_{0}\right) \cos \left(\alpha_{2} \xi_{0}\right)\right]}{D e t}$,

where Det $=-2 \alpha_{1} \alpha_{2}\left[\sinh ^{2}\left(\alpha_{1} \xi_{o}\right) \sin ^{2}\left(\alpha_{2} \xi_{o}\right)+\cosh ^{2}\left(\alpha_{1} \xi_{o}\right) \cos ^{2}\left(\alpha_{2} \xi_{o}\right)\right]$. By substituting the above $A_{1}$ and $B_{1}$ solutions into $Y_{1}^{\prime \prime \prime}\left(\xi_{o}\right)$ of Eq. (33) and applying the boundary condition of $Y_{1}^{\prime \prime \prime}\left(\xi_{0}\right)=0$, the following equation, which is also Eq. (20), is obtained after some manipulations

$\left[2 \alpha \sinh \left(\alpha_{1} \xi_{o}\right) \sin \left(\alpha_{2} \xi_{o}\right)+2 \sqrt{1-\alpha^{2}} \cosh \left(\alpha_{1} \xi_{o}\right) \cos \left(\alpha_{2} \xi_{o}\right)\right]\left[\frac{2\left[\cosh ^{2}\left(\alpha_{1} \xi_{o}\right)+\cos ^{2}\left(\alpha_{2} \xi_{o}\right)\right]}{\operatorname{Det}}+\frac{1}{2}\left(\frac{\alpha_{2}}{\alpha_{1}}+\frac{\alpha_{1}}{\alpha_{2}}\right)\right]$

$-\frac{8 \sqrt{1-\alpha^{2}}}{\text { Det }} \cosh \left(\alpha_{1} \xi_{o}\right) \cos \left(\alpha_{2} \xi_{o}\right)=0$.

Eq. (36), which is independent of the concentrated load $F$, is the equation to determine the separation point of $\xi_{0}$.

Once $\xi_{o}$ is determined by Eq. (36) and substituted into Eq. (35), $A_{1}$ and $B_{1}$ are found. Now only four unknowns of $A_{2}, B_{2}, C_{2}$ and $D_{2}$ are left, the transversality conditions of Eq. (13) provide four equations to solve these four unknowns.

For all other cases with different $\alpha$ values, the nine unknowns can also be determined by repeating the above three steps. For the succinctness purpose, we only summarize the results as follows.

Case I. $\alpha=0$

The separation point is determined as $\xi_{o}=\pi / 2$ by Eq. (19). The other eight unknowns are given as follows

$A_{1}=-\frac{F}{2} \operatorname{coth}\left(\frac{\pi}{2}\right), B_{1}=-A_{1}, H=-\frac{F}{2}, G=-H, A_{2}=B_{2}=0, C_{2}=-\frac{F}{\sinh \left(\frac{\pi}{2}\right)}, D_{2}=-\frac{\pi}{2} C_{2}$.

Case II. $0<\alpha<1$

The separation point of $\xi_{o}$ is determined as by Eq. (20) or the same equation as the above Eq. (36). The other eight unknowns are given as follows

$$
A_{1}=\frac{F\left(\alpha_{1} s h c h+\alpha_{2} s c\right)}{D e t}, B_{1}=\frac{F\left(-\alpha_{2} s h c h+\alpha_{1} s c\right)}{D e t}, H=-\frac{F}{2 \alpha_{1}}, G=\frac{F}{2 \alpha_{2}}, A_{2}=B_{2}=0,
$$

$C_{2}=A_{1}\left(\alpha_{1} \operatorname{chs}+\alpha_{2} \operatorname{sh} c\right)+B_{1}\left(\alpha_{1} \operatorname{sh} c-\alpha_{2} \operatorname{chs}\right)-\frac{F}{2 \alpha_{1}}\left(\alpha_{1} \operatorname{ch} c-\alpha_{2} s h s\right)+\frac{F}{2 \alpha_{2}}\left(\alpha_{1} \operatorname{sh} s+\alpha_{2} c h c\right)$,

$$
D_{2}=-\xi_{o} C_{2} \text {, }
$$

where $\alpha_{1}=\sqrt{1+\alpha}, \alpha_{2}=\sqrt{1-\alpha}$, Det $=-2 \alpha_{1} \alpha_{2}\left[\sinh ^{2}\left(\alpha_{1} \xi_{0}\right) \sin ^{2}\left(\alpha_{2} \xi_{0}\right)+\cosh ^{2}\left(\alpha_{1} \xi_{0}\right) \cos ^{2}\left(\alpha_{2} \xi_{o}\right)\right]$. Here for brevity reason, we define $\operatorname{sh}=\sinh \left(\alpha_{1} \xi_{0}\right)$, $c h=\cosh \left(\alpha_{1} \xi_{o}\right), s=\sin \left(\alpha_{2} \xi_{o}\right)$ and $c=\cos \left(\alpha_{2} \xi_{o}\right)$.

Case III. $\alpha=1$

The separation point of $\xi_{o}$ is determined as by Eq. (22). The other eight unknowns are given as follows 
$A_{1}=-\frac{F \sinh \left(\sqrt{2} \xi_{0}\right)}{2 \cosh \left(\sqrt{2} \xi_{0}\right)}, B_{1}=-\frac{F}{4 \cosh ^{2}\left(\sqrt{2} \xi_{0}\right)}\left[\sqrt{2} \sinh \left(\sqrt{2} \xi_{o}\right) \cosh \left(\sqrt{2} \xi_{o}\right)-2 \xi_{o}\right], H=-\frac{F}{2 \sqrt{2}}, G=\frac{F}{2}$,

$A_{2}=B_{2}=0, C_{2}=A_{1}\left[\sinh \left(\sqrt{2} \xi_{o}\right)+\sqrt{2} \xi_{o} \cosh \left(\sqrt{2} \xi_{o}\right)\right]+\sqrt{2} B_{1} \sinh \left(\sqrt{2} \xi_{o}\right)+\frac{\sqrt{2} F}{2} \xi_{o} \sinh \left(\sqrt{2} \xi_{o}\right), D_{2}=-\xi_{o} C_{2}$.

Case IV. $\alpha>1$

The separation point of $\xi_{o}$ is determined as by Eq. (23). The other eight unknowns are given as follows

$A_{1}=\frac{F\left(\gamma_{1}^{2}-\gamma_{2}^{2}\right)}{2 \gamma_{1} \sqrt{\alpha^{2}-1} D e t_{1}} \sinh \left(\gamma_{1} \xi_{o}\right) \cosh \left(\gamma_{2} \xi_{o}\right), B_{1}=\frac{F\left(\gamma_{2}^{2}-\gamma_{1}^{2}\right)}{2 \gamma_{1} \sqrt{\alpha^{2}-1} D_{t} \gamma_{1}} \frac{\gamma_{1}}{\gamma_{2}} \cosh \left(\gamma_{1} \xi_{o}\right) \sinh \left(\gamma_{2} \xi_{o}\right)$,

$H=\frac{F}{2 \gamma_{1} \sqrt{\alpha^{2}-1}}, G=-\frac{\gamma_{1}}{\gamma_{2}} H, A_{2}=B_{2}=0$,

$C_{2}=A_{1} \gamma_{1} \sinh \left(\gamma_{1} \xi_{o}\right)+B_{1} \gamma_{2} \sinh \left(\gamma_{2} \xi_{o}\right)+H \gamma_{1}\left[\cosh \left(\gamma_{1} \xi_{o}\right)-\cosh \left(\gamma_{2} \xi_{o}\right)\right], D_{2}=-\xi_{o} C_{2}$,

where $\gamma_{1}=\sqrt{2 \alpha+2 \sqrt{\alpha^{2}-1}}, \gamma_{2}=\sqrt{2 \alpha-2 \sqrt{\alpha^{2}-1}}$ and $\operatorname{Det}_{1}=\left(\gamma_{2}^{2}-\gamma_{1}^{2}\right) \cosh \left(\gamma_{1} \xi_{0}\right) \cosh \left(\gamma_{2} \xi_{0}\right)$.

Case V. $-1<\alpha<0$ The separation point of $\xi_{0}$ is determined as by Eq. (24). The other eight unknowns are given as follows

$A_{1}=\frac{F\left[\alpha_{1}^{*} s h c h+\alpha_{2}^{*} s c\right]}{D e t^{*}}, B_{1}=\frac{F\left[-\alpha_{2}^{*} s h c h+\alpha_{1}^{*} s c\right]}{D e t^{*}}, H=-\frac{F}{2 \alpha_{1}^{*}}, G=\frac{F}{2 \alpha_{2}^{*}}, A_{2}=B_{2}=0$,

$C_{2}=A_{1}\left(\alpha_{1}^{*} \operatorname{chs}+\alpha_{2}^{*} \operatorname{shc}\right)+B_{1}\left(\alpha_{1}^{*} \operatorname{sh} c-\alpha_{2}^{*} \operatorname{ch} s\right)-\frac{F}{2 \alpha_{1}^{*}}\left(\alpha_{1}^{*} \operatorname{ch} c-\alpha_{2}^{*} \operatorname{sh} s\right)+\frac{F}{2 \alpha_{2}^{*}}\left(\alpha_{1}^{*} \operatorname{sh} s+\alpha_{2}^{*} \operatorname{ch} c\right)$,

$D_{2}=-\xi_{0} C_{2}$,

where $\alpha_{1}^{*}=\sqrt{1-|\alpha|}, \quad \alpha_{2}^{*}=\sqrt{1+|\alpha|}$ and $\operatorname{Det}^{*}=-2 \alpha_{1}^{*} \alpha_{2}^{*}\left[\sinh ^{2}\left(\alpha_{1}^{*} \xi_{0}\right) \sin ^{2}\left(\alpha_{2}^{*} \xi_{0}\right)+\cosh ^{2}\left(\alpha_{1}^{*} \xi_{0}\right) \cos ^{2}\left(\alpha_{2}^{*} \xi_{0}\right)\right]$. Again, for brevity reason, we define $\operatorname{sh}=\sinh \left(\alpha_{1}^{*} \xi_{o}\right)$, ch $=\cosh \left(\alpha_{1}^{*} \xi_{o}\right), s=\sin \left(\alpha_{2}^{*} \xi_{o}\right)$ and $c=\cos \left(\alpha_{2}^{*} \xi_{o}\right)$.

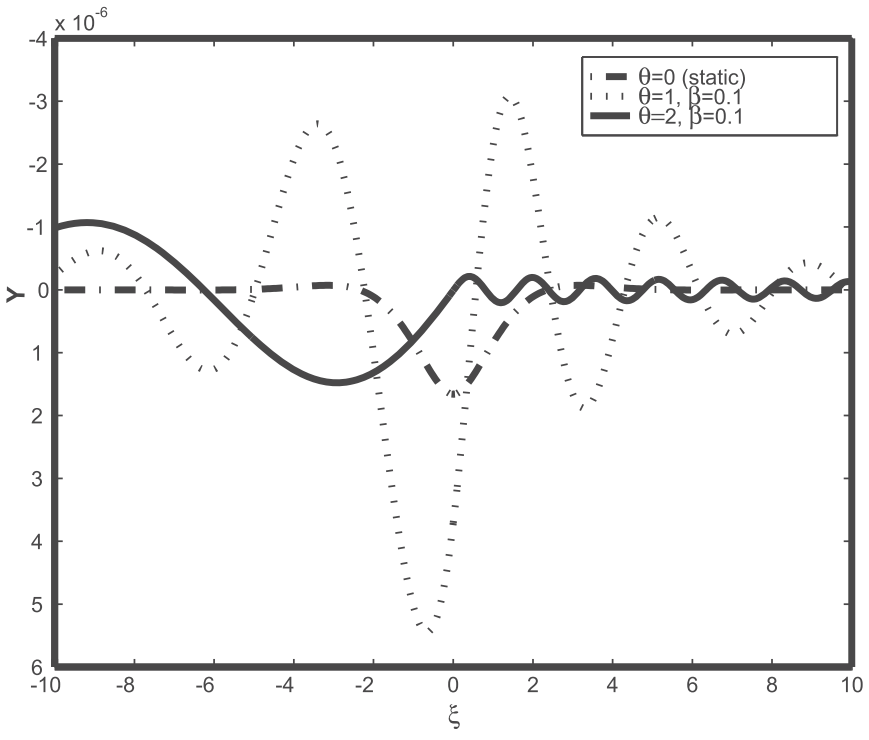

Fig. A1. The asymmetric wave shapes of a beam under a moving concentrated load with very high speeds. 


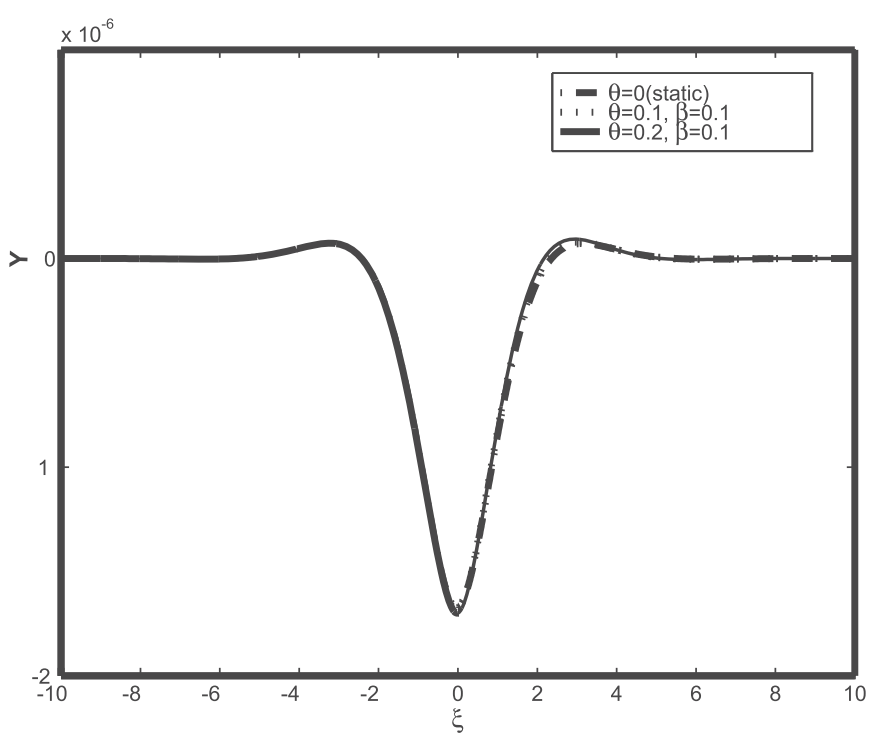

Fig. A2. The wave shapes of a beam under a moving concentrated load with low speeds.

\section{References}

Ang, K.K., Dai, J., 2013. Response analysis of high-speed rail system accounting for abrupt change of foundation stiffness. J. Sound Vib. 332, 2954-2970.

Ansari, M., Esmailzadeh, E., Younesian, D., 2010. Internal-external resonance of beams on non-linear viscoelastic foundation traversed by moving load. Nonlinear Dynam. 61, 163-182.

Ansari, M., Esmailzadeh, E., Younesian, D., 2011. Frequency analysis of finite beams on nonlinear Kelvin-Voight foundation under moving loads. J. Sound Vib. 330, 1455-1471.

Attar, M., Karrech, A., Regenauer-Lieb, K., 2016. Non-linear analysis of beam-like structures on unilateral foundations: a lattice spring model. Int. J. Solids Struct. 88-89, 192-214.

Bhattiprolu, U., Davies, P., Bajaj, A.K., 2014. Static and dynamic response of beams on nonlinear viscoelastic unilateral foundations: a multimode approach. J. Vib. Acoust. 136, 031002

Bhattiprolu, U., Bajaj, A.K., Davies, P., 2016. Periodic response predictions of beams on nonlinear and viscoelastic unilateral foundations using incremental harmonic balance method. Int. J. Solids Struct. 99, 28-39.

Bian, N., Jiang, H., Cheng, C., Chen, Y., Chen, R., Jiang, J., 2014. Full-scale model testing on a ballastless high-speed railway under simulated train moving loads. Soil Dynam. Earthq. Eng. 66, 368-384.

Chen, J., Chen, Y.K., 2011. Steady state and stability of a beam on a damped tensionless foundation. Int. J. Non-Linear Mech. 46, 180-185.

Chen, Y.H., Huang, Y.H., Sun, C.T., 1997. Responses of an infinite Timoshenko beam on a viscoelastic foundation to a harmonic moving load. J. Sound Vib. 241, 809-824.

Choros, J., Adams, G.A., 1979. A steadily moving load on an elastic beam resting on a tensionless Winkler foundation. J. Appl. Mech. 46, 175-180.

Dempsey, J.P., Keer, L.M., Patel, N.B., Glasser, M.L., 1984. Contact between plates and unilateral supports. J. Appl. Mech. 55, 324-328.

Dimitrovová, Z., 2017. New semi-analytical solution for a uniformly moving mass on a beam on a two-parameter visco-elastic foundation. Int. J. Mech. Sci. 127, 142-162.

Dutta, S.C., Roy, R., 2002. A critical review on idealization and modeling for interaction among soil-foundation-structure system. Comput. Struct. 80, 1579-1594.

Hetényi, M., 1946. Beams on Elastic Foundation. The University of Michigan Press, Ann Arbor, MI.

Johnson, K.L., 1985. Contact Mechanics. Cambridge University Press, Cambridge, UK.

Keer, L.M., Dundurs, J., Tsai, K.C., 1972. Problems involving a receding contact between a layer and a half space. J. Appl. Mech. 39, 1115-1120.

Kenney, J.T., 1954. Steady-state vibrations of beam on elastic foundation for moving load. J. Appl. Mech. 21, 359-364.

Kerr, A.D., 1964. Elastic and viscoelastic foundation models. J. Appl. Mech. 31, 491-498.

Kerr, A.D., 1972. The continuously supported rail subjected to an axial force and a moving load. Int. J. Mech. Sci. 14, 71-78.

Kerr, A.D., 1974. The stress and stability analyses of railroad tracks. J. Appl. Mech. 41, 841-848.

Kerr, A.D., 1976. On the derivation of well posed boundary value problems in structural mechanics. Int. J. Solids Struct. 12, 1-11.

Kerr, A.D., El-Aini, Y.M., 1978. Determination of admissible temperature increases to prevent vertical track buckling. J. Appl. Mech. 45, 565-573.

Labra, J.J., 1975. An axially stressed railroad track on an elastic continuum subjected to a moving load. Acta Mech. 22, 113-129.

Lancioni, G., Lenci, S., 2010. Dynamics of a semi-infinite beam on unilateral springs: touch-down points motion and detached bubbles propagation. Int. J. Non-Linear Mech. 45, 876-887.

Lim, N.H., Park, N.H., Kang, Y.J., 2003. Stability of continuous welded rail track. Comput.
Struct. 81, 2219-2236.

Lin, L., Adams, G., 1987. Beam on tensionless elastic foundation. J. Eng. Mech. 113, 542-553.

Ma, X., Butterworth, J., Clifton, G., 2009a. Response of an infinite beam resting on a tensionless elastic foundation subjected to arbitrarily complex transverse loads. Mech. Res. Commun. 36, 818-825.

Ma, X., Butterworth, J., Clifton, G., 2009b. Static analysis of an infinite beam resting in a tensionless Pasternak foundation. Eur. J. Mech. A Solid. 28, 697-703.

Maheshwari, P., Chandra, S., Basudhar, P.K., 2004. Responses of beams on a tensionless extensible geosynthetic-reinforced earth bed subjected to moving loads. Comput. Geotech. 31, 537-548.

Nobili, A., 2012. Variational approach to beams resting on two-parameter tensionless elastic foundations. J. Appl. Mech. 79, 021010.

Nobili, A., 2013. Superposition principle for the tensionless contact of a beam resting on a Winkler or a Pasternak foundation. J. Eng. Mech. 139, 1470-1478.

Press, W.H., Flannery, B.P., Teukolsky, S.A., Vettering, W.T., 1986. Numerical Recipes. Cambridge University Press, Cambridge, UK.

Reissner, E., 1958. A note on deflection of plates on a viscoelastic foundation. J. Appl. Mech. 25, 144-145.

Sapountzakis, E.J., Kampitsis, A.E., 2011a. Shear deformable beam-column partially supported on tensionless three-parameter foundation. Arch. Appl. Mech. 81, 1833-1851.

Sapountzakis, E.J., Kampitsis, A.E., 2011b. Nonlinear response of shear deformable beams on tensionless nonlinear viscoelastic foundation under moving loads. J. Sound Vib. 330, 5410-5426.

Sapountzakis, E.J., Kampitsis, A.E., 2013. Inelastic analysis of beams on two-parameter tensionless elastoplastic foundation. Eng. Struct. 48, 389-409.

Timoshenko, S., Langer, B.F., 1932. Stresses in railroad track. J. Appl. Mech. 54, 277-302.

Tran, M.T., Ang, K.K., Luong, V.H., 2014. Vertical dynamic response of non-uniform motion of high-speed rails. J. Sound Vib. 333, 5427-5442.

Tsai, N.C., Westmann, R.E., 1967. Beams on tensionless foundation. J. Eng. Mech. 93, $1-12$.

Weitsman, Y., 1969. On the unbonded contact between plates and an elastic half space. J. Appl. Mech. 36, 505-509.

Weitsman, Y., 1970. On foundations that react in compression only. J. Appl. Mech. 37, 1019-1030.

Weitsman, Y., 1971. Onset of separation between a beam and a tensionless elastic foundation under a moving load. Int. J. Mech. Sci. 13, 707-711.

Weitsman, Y., 1972. A tensionless contact between a beam and an elastic half-space. Int. J. Eng. Sci. 10, 73-81.

Wright, H.D., 1995. Local stability of filled and encased steel section. J. Struct. Eng. 121, 1382-1388 1995.

Younesian, D., Hosseinkhani, Askari, H., Esmailzadeh, E., 2019. Elastic and viscoelastic foundations: a review on linear and nonlinear vibration modeling and applications. Nonlinear Dynam (in press).

Zhang, Y., 2008. Tensionless contact of a finite beam resting on Reissner foundation. Int. J. Mech. Sci. 50, 1035-1041.

Zhang, Y., Liu, X., Wei, Y., 2018. Response of an infinite beam on a bilinear elastic foundation: bridging the gap between the Winkler and tensionless foundation models. Eur. J. Mech. A Solid. 71, 394-403.

Zhang, Y., Murphy, K.D., 2004. Response of a finite beam in contact with a tensionless foundation under symmetric and asymmetric loading. Int. J. Solids Struct. 41, 6745-6758.

Zhang, Y., Murphy, K.D., 2012. Tensionless contact of a finite circular plate. Acta Mech. Sin. 28, 1374-1381.

Zhang, Y., Murphy, K.D., 2013. Tensionless contact of a finite beam: concentrated load inside and outside the contact zone. Acta Mech. Sin. 29, 836-839. 